\title{
The Relationship Between Patient Reported Dysphagia Symptom Severity and Swallowing Related Quality of Life in Patients with Neurological Disorders
}

\author{
Selen Serel Arslan (D), Numan Demir (D), A. Ayse Karaduman (D) \\ Hacettepe University, Faculty of Physical Therapy and Rehabilitation Ankara, Turkey \\ Correspondence Author: Selen Serel Arslan \\ E-mail: selen.serel@hacettepe.edu.tr \\ Received: $16.01 .2018 \quad$ Accepted: 08.03 .2018
}

\begin{abstract}
Objective: The aim of this study was to determine the relationship between patient reported dysphagia symptom severity and swallowing related quality of life (SRQOL) in patients with neurological disorders.

Methods: Patients with a diagnosis of neurological disorders who aged between 25 to 60 years were included. The dysphagia symptom severity was assessed with the Turkish Eating Assessment Tool (T-EAT-10). SRQOL was assessed by the Turkish version of the Swallow Quality of Life Questionnaire (T-SWAL-QOL).

Results: Eighty-four patients with neurological disorder were included. The mean T-EAT-10 score was $19.59 \pm 11.16(\min =0, \max =40)$, and the mean total score of T-SWAL-QOL was $50.63 \pm 23.52(\mathrm{~min}=0$, $\max =90)$. A negative, good, statistically significant correlation between T-EAT-10 and total T-SWAL-QOL was found $(\mathrm{p}<0.05)$.

Conclusion: Higher patient reported dysphagia symptom severity is associated with lower scores of SRQOL. Therefore, appropriate management strategies which also focus on patient reported symptoms may be necessary to minimize negative SRQOL issues of patients with neurogenic dysphagia.

Key words: Deglutition, deglutition disorders, neurogenic dysphagia, quality of life
\end{abstract}

\section{INTRODUCTION}

Neurological disorders cause a high prevalence of oropharyngeal dysphagia which has significant complications including malnutrition, dehydration, and recurrent pneumonia $(1,2)$. Dysphagia also affects general health and wellbeing of patients due to several reasons. First, difficulty in swallowing causes inability to eat and drink. Second, risk of coughing, choking, and vomiting due to swallowing difficulty may cause increased anxiety and decreased self-esteem (3). Third, mealtimes may become stressful and mealtime pleasure may decrease due to dysphagia $(4,5)$.

Swallowing related quality of life (SRQOL) assessments have been used as outcome measures of the effect of diseases and/ or treatments (6). Quality of life of patients with neurogenic dysphagia has been investigated in several studies (7-11). One study concluded that SRQOL is associated with degree of swallowing impairment which is based on the penetration aspiration severity of the patients with amyotrophic lateral sclerosis (10). This study shows only one aspect related to swallowing disorders and therefore the relationship with more comprehensive aspect of swallowing disorders in addition to airway safety would also be investigated.
Patient reported outcome measures become very important because they are directly obtained from the patient without any interpretation. These outcomes are found to be related to disease and treatment effects (12) and allow clinicians to implement patient feedback into clinical practice (13). Therefore, patient reported information regarding their symptoms (i.e, type, frequency, severity of symptoms), perceptions and treatment effects should be essential for high quality clinical care (14). Patient reported outcome measures in dysphagia rehabilitation are also critical to understand different aspects of swallowing disorders including physiological as well as psychosocial aspects (15). It was reported that patient reported dysphagia symptom severity help to identify patients with oropharyngeal dysphagia and are sensitive to differences in dysphagia severity level (16-19). Therefore, patient reported dysphagia symptom severity would provide a comprehensive aspect of swallowing impairment. The aim of the current investigation was to determine the relationship between patient reported dysphagia symptom severity and SRQOL in patients with neurological disorders. We hypothesized that SRQOL would decrease in patients who perceived higher dysphagia symptom severity. 


\section{METHODS}

Patients with a diagnosis of neurological disorders who were referred for the first time due to complaints about swallowing function, aged between 25 to 60 years, and being cognitively normal according to the Mini Mental State Examination (20) were included. Patients with neurological disorders who were clinically unstable, had abnormal cognitive function, and had no dysphagia symptoms were excluded from the study. This prospective study was approved by the Non-interventional Clinical Researches Ethics Board. The written and signed approval form was obtained from the patients.

Descriptive information including age, gender, height, weight, diagnosis and type of feeding was noted. The dysphagia symptom severity was assessed by the Turkish Eating Assessment Tool (T-EAT-10) (17). The T-EAT-10 has sufficient internal consistency, test-retest reliability, and criterion validity. It can be used to predict risk of aspiration in patients with neurological disorders, thereby help to identify and refer patients with dysphagia for further evaluation (21). Patients filled the T-EAT-10, and if they need any guidance in terms of meaning of the questions or scoring system, a physical therapist who specialized in dysphagia research helped. It has 10 questions and every question is scored on a 5 -point scale between 0 to 4 . The score 0 means 'no problem', and the score 4 indicates 'severe problem'. The scores of the 10 questions are summed to obtain a total score. A score of three or more from the instrument indicates being at risk of swallowing disorders (16). Patients with a T-EAT-10 score more than 15 were 2.4 times more likely to have airway aspiration (21). The administration of the T-EAT-10 takes less than 2 minutes.

SRQOL of the patients were questioned by the Turkish version of the Swallow Quality of Life Questionnaire (T-SWAL-QOL) (22). It is a 44-item questionnaire, and the completion of the instrument takes 20-30 minutes. T-SWAL-QOL has 10 subscales related to generic and SRQOL. The subscales related to generic quality of life include fatigue and sleep. The subscales related to SRQOL are burden, eating duration, eating desire, food selection, communication, fear, mental health and social functioning. There are two types of questions in the instrument. Patients are asked to define the frequency of a situation in some questions. The rest of questions focus on the degree of agreement of the items. Both frequency and evaluation questions are in the form of a 5 point Likert scale. All subscales range from 0 to 100 , the lower the score, the worse SRQOL. It was reported that global scores in SWALQOL is approximately 65-80 (10).

All patients completed the instruments in a quiet room.

\section{Statistical analysis}

The IBM-SPSS for Windows version 20 was used to perform statistical analysis. Number/percent for qualitative data and mean, standard deviation, minimum and maximum for quantitative data were used for descriptive statistics. The non-parametric Spearman correlation coefficient was used to assess correlation between quantitative variables. Correlation coefficients between $0.05-0.30$ show low correlation; where, $0.30-0.40$ show low to moderate correlation, $0.40-0.60$ show moderate correlation, $0.60-0.70$ show good correlation, $0.70-0.75$ show strong correlation, and $0.75-1.00$ show excellent correlation (23). The MannWhitney $U$ test was used to compare the patients according to the type of feeding. A p-value of less than 0.05 was considered as statistically significant.

\section{RESULTS}

Eighty-four patients with neurological disorder were included, of which $57.1 \%$ were female. The mean age, height and weight of the patients were $48.14 \pm 9.03$ years $(\mathrm{min}=25$, $\max =60), 162.56 \pm 9.85 \mathrm{~cm}(\min =145, \max =190)$, and 68.16 $\pm 12.82 \mathrm{~kg}(\min =45, \max =98)$, respectively. A percentage of 71.4 of the patients $(n=60)$ were stroke patients, $15.5 \%$ $(n=13)$ were patients with Parkinson's disease, and $13.1 \%$ $(n=11)$ were patients with Multiple sclerosis. A percentage of 58.3 of the patients $(n=49)$ were fed orally and $41.7 \%$ of them fed non-orally ( $n=35)$.

The mean T-EAT-10 score was $19.59 \pm 11.16$ ( $\min =0$, $\max$ $=40$ ), and the mean total score of T-SWAL-QOL was $50.63 \pm$ $23.52(\min =0, \max =90)$. The mean scores of the T-SWALQOL are shown in Table 1.

Table 1. The mean T-EAT-10 and T-SWAL-QOL scores

\begin{tabular}{llll}
\hline & $X$ & $S D$ & min-max \\
\hline T-EAT-10 & 19.59 & 11.16 & $0-40$ \\
\hline Total T-SWAL-QOL & 50.63 & 23.52 & $0-90$ \\
\hline Burden & 40.09 & 31.79 & $0-100$ \\
\hline Food selection & 64.79 & 30.86 & $0-100$ \\
\hline Eating duration & 64.76 & 32.23 & $0-100$ \\
\hline Eating desire & 54.29 & 28.31 & $0-100$ \\
\hline Fear & 55.96 & 33.71 & $0-100$ \\
\hline Sleep & 45.84 & 30.99 & $0-100$ \\
\hline Fatigue & 43.04 & 31.17 & $0-100$ \\
\hline Communication & 51.85 & 33.43 & $0-100$ \\
\hline Mental health & 51.33 & 37.17 & $0-100$ \\
\hline Social functioning & 41.39 & 34.22 & $0-100$ \\
\hline
\end{tabular}

A negative, good, statistically significant correlation between T-EAT-10 and total T-SWAL-QOL was detected $(p<0.001, r=$ $-0.69)$. The correlations between T-EAT-10 and the subscales of T-SWAL-QOL are displayed in Table 2. Worse scores in T-SWAL-QOL were associated with worse scores in T-EAT-10. 
Table 2. The correlation T-EAT-10 and T-SWAL-QOL scores

\begin{tabular}{lll} 
& & T-EAT-10 \\
& $r$ & $p$ \\
\hline T-SWAL-QOL total & $-0.69 * * * *$ & $\mathrm{p}<0.001$ \\
\hline Burden & $-0.39 * * *$ & $\mathrm{p}<0.001$ \\
Food selection & $-0.56 * * *$ & $\mathrm{p}<0.001$ \\
\hline Eating duration & $-0.28^{*}$ & 0.01 \\
\hline Eating desire & $-0.58^{* * *}$ & $\mathrm{p}<0.001$ \\
\hline Fear & $-0.57 * * *$ & $\mathrm{p}<0.001$ \\
\hline Sleep & $-0.49 * * *$ & $\mathrm{p}<0.001$ \\
Fatigue & $-0.37 * *$ & 0.001 \\
\hline Communication & $-0.34 * *$ & 0.002 \\
Mental health & $-0.67 * * * *$ & $\mathrm{p}<0.001$ \\
\hline Social functioning & $-0.59 * * *$ & $\mathrm{p}<0.001$ \\
\hline
\end{tabular}

* low correlation, $* *$ low to moderate correlation, $* * *$ moderate correlation, $* * * *$ strong correlation

A significant difference was found between patients according to the type of feeding in terms of the T-EAT-10 and T-SWAL-QOL $(p=0.01$ ) (Table 3).

Table 3. The T-EAT-1O and T-SWAL-QOL scores of patients according to the type of feeding

\begin{tabular}{llllll}
\hline & \multicolumn{3}{l}{$\begin{array}{l}\text { Patients who fed } \\
\text { orally }\end{array}$} & \multicolumn{3}{l}{ Patients who fed non-orally } \\
& X (SD) & min- & X (SD) & min-max & \\
T-EAT-10 & $16.77(9.70)$ & $0-36$ & $23.54(11.96)$ & $16.77(9.70)$ & 0.01 \\
T-SWAL-QOL & $55.85(20.9)$ & $13-90.7$ & $43.32(25.27)$ & $55.85(20.9)$ & 0.01 \\
\hline
\end{tabular}

\section{DISCUSSION}

In this study, we observed a relationship between patient reported dysphagia symptom severity and SRQOL. As hypothesized, the more patient reported dysphagia symptom severity, the worse the patient reported SRQOL.

Dysphagia is a common consequence of several neurologic diseases including stroke, multiple sclerosis, motor neuron disease, Parkinson's disease and myopathy due to sensorimotor impairment of the oral, pharyngeal and esophageal phases of swallowing (24-26). According to our study results, SRQOL is significantly reduced in patients with neurogenic dysphagia and the most affected subscales were burden, social functioning, fatigue and sleep. Literature defining SRQOL of patients with neurogenic dysphagia also points out that fatigue, eating duration, burden, and sleep were the mostly affected domains $(10,11,26)$. A study investigating the social and psychological burden of dysphagia reported that approximately one third of the patients avoided eating with other people indicating decreased socialization (27) as our study results. Therefore, timely and efficient swallowing evaluation become important to identify symptoms and plan appropriate dysphagia rehabilitation program in patients with neurogenic dysphagia for reducing these swallowing related consequences.
SRQOL was associated with patient reported dysphagia symptom severity. Greater degree of dysphagia symptom severity was found to be associated with lower scores of SRQOL, and lower degree of dysphagia symptom severity was associated with better SRQOL. In addition, patients who fed orally (better swallowing function) had better SRQOL than patients who fed nonorally (worse swallowing function). Patient reported dysphagia symptom severity determined by the T-EAT-10 provides a global perspective related to patients' swallowing impairment. In a previous study conducted with Amyotrophic Lateral Sclerosis showed the relationship between SRQOL and degree of airway safety which is one aspect of swallowing impairment (10). Our study results could be complementary to this study result. As well as airway safety which can be considered as an objective measure of pharyngeal phase swallowing impairment, perceived dysphagia symptoms from patients' point of view are also associated with the amount of affected SRQOL. Therefore, patient reported dysphagia symptom severity can not only provide information regarding disease severity but also can help document the effect on the patients' quality of life.

This current study provides information regarding the relationship between patient reported dysphagia symptom severity and SRQOL. As the main outcome measures of this study are both patient reported, an objective swallowing evaluation will also be added to support current findings.

\section{CONCLUSION}

In conclusion, patient reported dysphagia symptom severity was found to be associated with SRQOL. The results of this study highlight that appropriate management strategies which also focus on patient reported symptoms may be necessary to minimize negative SRQOL issues of patients with neurogenic dysphagia.

Conflict of Interest: The authors declare that they have no conflict of interest.

Financial Disclosure: No financial support.

\section{REFERENCES}

[1] Miles A, Allen JE. Management of oropharyngeal neurogenic dysphagia in adults. Curr Opin Otolaryngol Head Neck Surg 2013;23: 433-39.

[2] Campbell BH, Spinelli K, Marbella AM, Myers KB, Kuhn JC, Layde PM. Aspiration, weight loss and quality of life in head and neck cancer survivors. Arch Otolaryngol Head Neck Surg 2004; 130: 1100-03.

[3] Ekberg O, Hamdy S, Woisard V, Wuttge-Hannig A, Ortega P. Social and psychological burden of dysphagia: its impact on diagnosis and treatment. Dysphagia 2002; 17:139-46.

[4] Gustafsson B, Tibbling L. Dysphagia, an unrecognized handicap. Dysphagia 1991; 6: 193-99.

[5] McHorney CA, Martin-Harris B, Robbins J, Rosenbek J. Clinical validity of the SWAL-QOL and SWAL-CARE outcome tools with respect to bolus flow measures. Dysphagia 2006; 3:141-48. 
[6] Timmerman AA, Speyer R, Heijnen BJ, Klijn-Zwijnenberg IR. Psychometric characteristics of health-related quality-of-life questionnaires in oropharyngeal dysphagia. Dysphagia 2014; 29:183-98.

[7] Miller N, Noble E, Jones D, Burn D. Hard to swallow: dysphagia in Parkinson's disease. Age Ageing 2006; 35: 614-18.

[8] de Luis DA, Izaola O, de Mateo ML, Cuellar L, Terroba MC, Aller R. Quality of life, dietary intake in elderly patients with dysphagia. Nutrition 2006; 22: 584.

[9] Paris G, Martinaud O, Petit A, Cuvelier A, Hannequin D, Roppeneck $P$, Verin E. Oropharyngeal dysphagia in amyotrophic lateral sclerosis alters quality of life. J Oral Rehabil 2013; 40:199-204.

[10] Tabor L, Gaziano J, Watts S, Robison R, Plowman EK. Defining swallowing-related quality of life profiles in individuals with Amyotrophic Lateral Sclerosis. Dysphagia 2006; 31:376-82.

[11] Leow LP, Huckabee ML, Anderson T, Beckert L. The Impact of dysphagia on quality of life in ageing and Parkinson's disease as measured by the Swallowing Quality of Life (SWAL-QOL) Questionnaire. Dysphagia 2010; 25:216-20.

[12] Deshpande PR, Rajan S, Sudeepthi BL, Abdul Nazir CP. Patientreported outcomes: A new era in clinical research. Perspect Clin Res 2011; 2:137-44.

[13] Holmes MM, Lewith G, Newell D, Fiend J, Bishop FL. The impact of patient-reported outcome measures in clinical practice for pain: a systematic review. Qual Life Res. 2017; 26(2):245-57.

[14] Weldring T, Smith SMS. Patient-Reported Outcomes (PROs) and Patient-Reported Outcome Measures (PROMs). Health Serv Insights. 2013; 6:61-68.

[15] Patel DA, Sharda R, Hovis KL, Nichols EE, Sathe N, Penson $D F$, Feurer ID, McPheeters ML, Vaezi MF, Francis DO. Patientreported outcome measures in dysphagia: a systematic review of instrument development and validation. Dis Esophagus. 2017; 30(5):1-23.

[16] Belafsky PC, Mouadeb DA, Rees CJ, Pryor JC, Postma GN, Allen J, Leonard RJ.Validity and reliability of the Eating Assessment Tool (EAT-10). Ann Otol Rhinol Laryngol 2008; 117:919-24.
[17] Demir N, Serel Arslan S, İnal Ö, Karaduman AA. Relability and validity of the Turkish Eating Assessment Tool (T-EAT-10). Dysphagia 2016; 31:644-9.

[18] Wallace KL, Middleton S, Cook IJ. Development and validation of a self-report symptom inventory to assess the severity of oral-pharyngeal dysphagia. Gastroenterology 2000; 118:67887.

[19] Grudell AB, Alexander JA, Enders FB, Pacifico R, Fredericksen $M$, Wise JL, Locke GR 3rd, Arora A, Zais T, Talley NJ, Romero Y. Validation of the Mayo Dysphagia Questionnaire. Dis Esophagus 2007; 20:202-05.

[20] Tombaugh TN, Mclntyre NJ. The mini-mental State Examination: A comprehensive review. J Am Geriatr Soc 1992; 40:922-35.

[21] Serel Arslan S, Demir N, Kılınç HE, Karaduman AA. The ability of the Eating Assessment Tool-10 to detect aspiration in patients with neurological disorders. J Neurogastroenterol Motil. 2017; 23(4):550-4.

[22] Demir N, Serel Arslan S, İnal Ö, Ünlüer NÖ, Karaduman AA. Reliability and validity of the Turkish version of the Swallow Quality Of Life Questionnaire. Turk J Physiother Rehabil 2016;27:19-24.

[23] Mukaka MM. Statistics Corner: A guide to appropriate use of Correlation coe cient in medical research. Malawi Med J 2012; 24:69-71.

[24] Buchholz DW. Dysphagia associated with neurological disorders. Acta Otorhinolaryngol Belg. 1994; 48(2):143-55.

[25] Patti F, Emmi N, Restivo DA, Liberto A, Pappalardo A, Torre LM, Reggio A.Neurogenic dysphagia: physiology, physiopathology and rehabilitative treatment. Clin Ter. 2002; 153(6):403-9.

[26] Gaspar M, Pinto GS, Gomes RHS, Santos RS, Leonor VD. Evaluation of quality of life in patients with neurogenic dysphagia. Revista CEFAC 2015; 17:1939-45.

[27] Ekberg O, Hamdy S, Woisard V, Wuttge-Hannig A, Ortega P. Social and psychological burden of dysphagia: Its impact on diagnosis and treatment. Dysphagia 2002; 17:139-46. 